\title{
Diagnostic utility of endobronchial ultrasound features in differentiating malignant and benign lymph nodes
}

\author{
Sumita Agrawal1, Pranav Ish1, Akhil Dhanesh Goel2, Nitesh Gupta1, Shibdas Chakrabarti', \\ Dipak Bhattacharya1, Manas Kamal Sen1, Jagdish Chander Suri1 \\ 1 Department of Pulmonary, Critical Care \& Sleep Medicine, Vardhaman Mahavir Medical College \\ and Safdarjung Hospital, New Delhi \\ 2 Department of Community Medicine and Family Medicine, All India Institute of Medical Sciences, \\ Jodhpur, India
}

\begin{abstract}
Endobronchial ultrasound (EBUS) features have been shown to be useful in predicting aetiology of enlarged malignant lymph nodes. However, there is dearth of evidence especially from developing countries. We assessed the EBUS characteristics across various mediastinal and hilar lymphadenopathies. In this prospective study, all patients with mediastinal and hilar lymphadenopathy on CT Chest and who were planned for EBUS-FNA (Fine Needle Aspiration) were included. EBUS features of lymph nodes studied were shape, size, margins, echogenicity, central hilar structure (CHS), coagulation necrosis sign and colour power doppler index (CPDI). These were scored and compared between benign and malignant lymphadenopathies. A total of 86 lymph nodes in 46 patients were prospectively studied of which 23 (26.7\%) were malignant, 27 (31.3\%) tuberculosis and 36 (41.8\%) sarcoidosis. There was significant difference between malignant and benign lymph nodes in terms of CHS (central hilar structure)
\end{abstract}

Corresponding author: Dr. Sumita Agrawal, Dept. of Pumonary, Critical Care and Sleep Medicine, Safdarjung Hospital, Ward 32, third floor, Old Casualty Building, New Delhi 110029, India.

Mobile: +91.9643158274. E-mail: drsumi84@gmail.com.

Key words: EBUS; coagulation necrosis sign; lung cancer; sensitivity.

Contributions: SA, ADG, SC, DB, MKS, JCS, conception / design of the work; SA, PI, SC, DB, MKS, JCS, data acquisition; SA, ADG, JCS, data analysis; SA, PI, ADG, NG, JCS, data interpretation. All author draft / revised the work for important intellectual content, approved the version to be published.

Disclosure: Part of these findings were presented as a poster at ATS 2017 in Washington DC, by Dr. Sumita Agrawal.

Received for publication: 23 February 2018

Accepted for publication: 3 June 2018

(C) Copyright S. Agrawal et al., 2018

Tipografia PI-ME Editrice, Italy

Monaldi Archives for Chest Disease 2018; 88:928

doi: 10.4081/monaldi.2018.928

This article is distributed under the terms of the Creative Commons Attribution Noncommercial License (by-nc 4.0) which permits any noncommercial use, distribution, and reproduction in any medium, provided the original author(s) and source are credited. $(p=0.011)$, margins $(p=0.036)$ and coagulation necrosis sign $(\mathrm{p}<0.001)$. On comparison of features of malignancy and tuberculosis, there were significant differences in margins $(\mathrm{p}=0.016)$ and coagulation necrosis sign $(\mathrm{p}<0.001)$. However, when malignancy and sarcoidosis was compared, there were differences in echogenicity $(p=0.002)$, CHS $(p=0.009)$ and coagulation necrosis sign $(p<0.001)$. Only coagulation necrosis sign was found to be highly consistent with malignant lymph nodes. The other features cannot be used to distinguish malignant from benign lymph nodes, especially in a developing country like India where tuberculosis is a common cause of mediastinal lymphadenopathy.

\section{Introduction}

With the high prevalence of infectious and inflammatory conditions in India, CT (Computed Tomography) or PET-CT (Positron Emission Tomography-CT) alone may be inconclusive in differentiating benign from malignant lymph nodes (LN) in a substantial proportion of patients [1]. Due to these limitations of radiological diagnosis a tissue sample is needed for confirmation by either histopathology or cytopathology [1].

The broad application of Endobronchial Ultrasound (EBUS) has opened new opportunities for ultrasound guided lymph node sampling [2]. It has emerged as a safe method to obtain tissue from mediastinum, hilar or lesions in close proximity to central airways, showing an accuracy of $80-90 \%$ and complication incidence of less than 1\% [3]. Several studies have also shown that sonographic findings seem to be associated with malignancy in head and neck, breast, cervix or oesophageal cancer and the characteristics as seen on ultrasound can be used to predict the aetiology of these lesions [4,5]. It has been observed that sonographic analysis of cervical lymph nodes features like round shape, sharp borders, absence of central hilus, coagulation necrosis, heterogeneous echogenicity, increased peripheral vascularity are associated with metastasis. However, it has been documented that many of these features can overlap with tuberculosis [4]. Hence, in a developing country like India where tuberculosis is prevalent, it is important to distinguish between malignant and benign nodes as the management and prognosis differs.

Fujiwara et al. in 2010 studied the ultrasound features during EBUS in lung cancer patients and found that these characteristics can be used to categorize lymph nodes as malignant and benign [6]. In literature, revalidation of these scores has been done and a composite score has been designed [7,8]. However, none of these studies had included benign cases like tuberculosis which is more prevalent in developing countries and most of them were retrospective studies. In 
this study we have tried to explore the utility of sonographic features of mediastinal lymph nodes to differentiate between malignant and benign aetiologies.

\section{Materials and Methods}

This was a prospective study performed at a tertiary care centre over a period of one year. During the study period of one year, consecutive patients indicated for EBUS were enrolled. The institutional ethical committee had approved the protocol.

All patients above 18 years of age presenting with respiratory complaints, and an indication for EBUS-TBNA were eligible for inclusion in the study. Exclusion criteria were absolute contraindications to EBUS like severe hypoxemia, uncorrectable coagulopathy, life threatening cardiac arrhythmias or relative contraindications like recent myocardial infarction, pulmonary hypertension, uraemia and unstable asthma.

\section{Study procedure}

A written informed valid consent was taken from all patients. They were interviewed for demographic details and clinical history using a structured questionnaire. EBUS was performed using a convex probe EBUS Olympus-BF-UC180F EBUS scope and the EU-ME2 ultrasound (Olympus). For TBNA, a 22-gauge needle was used (Olympus). The data collected included age, gender, symptoms, signs, CT scan findings, group of lymph nodes involved and USG (ultrasonographic) features.

All procedures were performed by two pulmonologist, a nursing staff and were done under conscious sedation with a mouth guard using intravenous midazolam and fentanyl as advised by the BTS guidelines for bronchoscopy [9]. Local anaesthesia was achieved by spraying $10 \%$ lignocaine in the posterior pharynx prior to the procedure. The bronchoscope was passed by one bronchoscopist via the mouth guard. The vocal cords, trachea and carina were visualised. The sampling procedure followed the usual standard wherein the highest station is sampled first and then progressively downwards. As the lymph nodes were visualised in this order, their features were also studied and this order and then sampled. The number of lymph nodes sampled per patients ranged from 1-3 depending on which station nodes were enlarged and their accessibility. The features did not influence the choice of sampling. Enlarged lymph nodes were identified using digitally captured ultrasound images. Doppler applied to identify vessels and features studied and noted. The ultrasound score for each lymph node was given by the two pulmonologists separately before performing ROSE (rapid onsite evaluation). The highest level of node visualised were sampled first and assessed by a pathologist by ROSE. In case, diagnosis on ROSE was not achieved at this level, the next lower level of lymph nodes were sampled [10]. The 22-gauge Olympus needle was used to puncture the lymph nodes and after each pass, slides were prepared. The pathologist stained them with toluidine blue to identify lymphocytes, granulomas and atypical cells. Up to three passes were made per lymph node station until onsite confirmation by pathologist. The slides were then air dried and sent for cytopathological analysis and acid-fast bacilli stain. In all cases, material was also obtained for mycobacterial culture and malignant cell cytology. The final pathological report was then obtained and taken as gold standard.

Diagnosis of tuberculosis was made on the basis of clinical context plus presence of caseous granuloma or necrosis or acid fast bacillus (AFB) stain or culture. Diagnosis of sarcoidosis was made on the basis of clinical context plus non caseating granulomas. Diagnosis of lung cancer was made on the basis of atypical cells, pathological features suggestive of non-small cell/ small cell carcinoma and/or immunohisto- chemistry. Diagnosis of lymphoma was made on basis of atypical lymphocytes and immunohistochemistry.

Others were based on specific findings of the disease.

\section{Description of EBUS features}

The following sonographic features were evaluated:

1. Shape: round $v s$ oval; round defined as a ratio of $<1.5$ between 2 perpendicular axes.

2. Margins: distinct $v s$ indistinct. Distinct margins defined as well defined borders distinguished by a marked white line delimiting the LN.

3. Size: small axis $>10 \mathrm{~mm} v s<10 \mathrm{~mm}$.

4. Echogenicity: heterogeneous vs homogeneous.

5. Central Hilar Structure (CHS) in the form of a central linear structure with high echogenicity, with or without a blood vessel: present vs absent.

6. Colour Power Doppler Index (CPDI): Power Doppler mode was used to grade the blood flow in LN. The amount of blood flow was classified from 0 to 3 : grade 0 , no blood flow; grade 1, small amount of flow, one or two punctiform or short rod-shapes of colour flow signal; grade 2, medium amount of flow, one main vessel or a few small vessels could be found as a long strip of a curve; grade 3 , rich flow. CPDI was used as per Schmid Bindert et al. study [7].

7. Coagulation Necrosis Sign: it is a hypoechoic area within the lymph node without blood flow.

Shafiek et al. has proposed a scoring of the EBUS features as a method of predicting malignancy [8]. The same scoring system was used in this study to characterise the lymphnodes:

1. Heterogeneous (1.5 point) $v$ s homogeneous ( 0 point) echogenicity.

2. Absence of CHS (1.5 point) vs presence ( 0 point).

3. Round shape (1 vs 0 point).

4. Small $L N$ axis $\geq 10 \mathrm{~mm}$ (1 point).

5. Well-defined LN margin (1 point) $v s$ indistinct margin ( 0 point).

6. CPDI (0 point) for grades 0 to 1 vs (1 point) for grades 2 and 3.

The study of features and scoring were done as per previous similar studies by Fujiwara et al. [11], Shafiek et al. [8] and Schmid-Bindert et al. [7]. These features were studied by the pulmonologists performing the procedure. The cytology samples were reported by the pathologist remaining unaware of the sonography features.

\section{Data analysis}

SPSS (Statistical Package for Social Sciences) 20 program for Windows was used for statistical analysis. All the qualitative data was expressed as frequency and percentage and the quantitative data was expressed as a mean and standard deviation. Diagnostic accuracy of various EBUS features was assessed using sensitivity, specificity for discriminative ability. Positive and Negative predictive value for post-test probability of disease were also calculated. Unpaired $t$-test was used for quantitative data comparison. Differences were considered significant when $\mathrm{p}<0.05$.

\section{Results}

A total of 46 patients were recruited during the one-year study period from April 2016 to March 2017. The age ranged from 17 to 70 years with $53.5 \%$ males and $46.5 \%$ females. Patients with malignancy were older $(57.72 \pm 7.32)$ that those with tuberculosis $(34.04 \pm 15.16)$ or sarcoidosis $(42.10 \pm 8.91)$. Similarly, patients with malignancy had a higher proportion of smokers (65.2\%) as compared to benign patients (Table 1). 
Of the 86 lymph nodes in these 46 patients, majority of diagnosis was made at level $4 R(n=27,31.4 \%)$ and level $7(n=48,55.8 \%)$. Four patients also had level 11 nodes on CT scan but in all of these diagnoses could be made at higher level and hence they were not explored. Of the 86 lymph nodes, 23 (26.7\%) were malignant, 27 (31.3\%) were tuberculosis and 36 (41.8\%) were sarcoidosis.

Malignant nodes were more likely to show absence of central hilar structure $(p=0.011)$, distinct margins $(p=0.036)$ and coagulation necrosis sign $(p<0.001)$. Tuberculous nodes were significantly less likely to have distinct margins $(\mathrm{p}=0.016)$ or coagulation necrosis sign $(\mathrm{p}<0.001)$ as compared to malignant nodes. Similarly, sarcoid nodes are significantly less likely to have heterogenous echogenicity $(\mathrm{p}=0.002)$, absence of central hilar structures $(\mathrm{p}=0.009)$ and presence of coagulation necrosis sign ( $\mathrm{p}<0.001)$ (Table 2$)$.

Presence of coagulation necrosis sign was highly sensitive and specific for malignancy with positive predictive value of $80.7 \%$ and negative predictive value of $96.11 \%$ and a diagnostic accuracy of $91.86 \%$ (Table 3 ). Heterogenous echogenicity, absence of CHS and distinct margins although have low diagnostic accuracy but have a high negative predictive value of $84 \%$, $85.7 \%$ and $88.5 \%$ (Table 3). The scoring pattern as suggested by Shafiek et $a l$. had an AUC $=0.775$ (95\%CI: 0.68-0.89). Addition of radiological features of CPDI and coagulation necrosis factor improved the scoring pattern to an AUC $=0.862$ (95\%CI: 0.78-0.94). There was no significant difference between the diagnostic accuracy of the 2 scoring patterns (Figure 1).

Table 1. Patient characteristics and demographic profile.

\begin{tabular}{|c|c|c|c|c|c|}
\hline & $\begin{array}{c}\text { Total } \\
(n=86)\end{array}$ & $\begin{array}{c}\text { Malignancy } \\
\quad(n=23)\end{array}$ & $\begin{array}{l}\text { Benign } \\
(n=63)\end{array}$ & $\begin{array}{l}\text { Tuberculosis } \\
\qquad(n=27)\end{array}$ & $\begin{array}{c}\text { Sarcoidosis } \\
(n=36)\end{array}$ \\
\hline Age & $44.2 \pm 14.0$ & $57.1 \pm 7.8$ & $39.5 \pm 12.8$ & $34.04 \pm 15.16$ & $42.10 \pm 8.91$ \\
\hline \multicolumn{6}{|l|}{ Gender } \\
\hline Female & $38(44.2 \%)$ & $4(17.4 \%)$ & $34(54.0 \%)$ & $14(51.9 \%)$ & $20(55.6 \%)$ \\
\hline Male & $48(55.8 \%)$ & $19(82.6 \%)$ & $29(46.0 \%)$ & $13(48.1 \%)$ & $16(44.4 \%)$ \\
\hline \multicolumn{6}{|l|}{ Smoker } \\
\hline No & 67 (77.9\%) & $7(30.4 \%)$ & $60(95.2 \%)$ & $27(100.0 \%)$ & $33(91.7 \%)$ \\
\hline Yes & $19(22.1 \%)$ & $16(69.6 \%)$ & $3(4.8 \%)$ & $0(0.0 \%)$ & $3(8.3 \%)$ \\
\hline \multicolumn{6}{|c|}{ Lymph node level of EBUS } \\
\hline $10 \mathrm{~L}$ & $1(1.2 \%)$ & $1(4.3 \%)$ & $0(0.0 \%)$ & $0(0.0 \%)$ & $0(0.0 \%)$ \\
\hline $10 \mathrm{R}$ & $5(5.8 \%)$ & $1(4.3 \%)$ & $4(6.3 \%)$ & $2(7.4 \%)$ & $2(5.6 \%)$ \\
\hline $2 \mathrm{R}$ & $3(3.5 \%)$ & $1(4.3 \%)$ & $2(3.2 \%)$ & $0(0.0 \%)$ & $2(5.6 \%)$ \\
\hline $4 \mathrm{~L}$ & $3(3.5 \%)$ & $2(8.7 \%)$ & $1(1.6 \%)$ & $0(0.0 \%)$ & $1(2.8 \%)$ \\
\hline $4 \mathrm{R}$ & $27(31.4 \%)$ & $7(30.4 \%)$ & $20(31.7 \%)$ & $10(37.0 \%)$ & $10(27.8 \%)$ \\
\hline 7 & $47(54.7 \%)$ & $11(47.8 \%)$ & $36(57.1 \%)$ & $15(55.6 \%)$ & $21(58.3 \%)$ \\
\hline
\end{tabular}

Table 2. Distribution of EBUS features in malignant and benign lymph nodes.

\begin{tabular}{|c|c|c|c|c|c|c|c|}
\hline \multirow[b]{2}{*}{ EBUS features } & \multirow{2}{*}{$\begin{array}{c}\text { Malignancy } \\
\begin{array}{c}(\mathrm{n}=23) \\
\mathrm{Col} \%\end{array}\end{array}$} & \multicolumn{2}{|c|}{$\begin{array}{l}\text { Benign } \\
(\mathrm{n}=63)\end{array}$} & \multicolumn{2}{|c|}{$\begin{array}{l}\text { Tuberculosis } \\
\qquad(n=27)\end{array}$} & \multicolumn{2}{|c|}{$\begin{array}{l}\text { Sarcoidosis } \\
\quad(n=36)\end{array}$} \\
\hline & & $\mathrm{Col} \%$ & p value* & $\mathrm{Col} \%$ & p value $^{\circ}$ & $\mathrm{Col} \%$ & p value \# \\
\hline Heterogenous echogenicity & $65.2 \%$ & $33.3 \%$ & $0.080 \mathrm{a}$ & $44.4 \%$ & $0.142^{\mathrm{a}}$ & $25.0 \%$ & $0.002^{a}$ \\
\hline Round shape & $78.3 \%$ & $79.4 \%$ & $1.000 \mathrm{~b}$ & $92.6 \%$ & $0.225^{b}$ & $69.4 \%$ & $0.458 \mathrm{a}$ \\
\hline Absence of CHS & $73.9 \%$ & $42.9 \%$ & $0.011^{\mathrm{a}}$ & $48.1 \%$ & $0.064 \mathrm{a}$ & $38.9 \%$ & $0.009 \mathrm{a}$ \\
\hline Distinct margins & $87.0 \%$ & $63.5 \%$ & $0.036^{\mathrm{a}}$ & $55.6 \%$ & $0.016^{\mathrm{a}}$ & $69.4 \%$ & $0.123^{a}$ \\
\hline Small axis diameter $>10 \mathrm{~mm}$ & $91.3 \%$ & $92.1 \%$ & $1.000^{b}$ & $100.0 \%$ & $0.207 \mathrm{~b}$ & $86.1 \%$ & $0.694 \mathrm{~b}$ \\
\hline CPDI index 1 & $65.2 \%$ & $54.0 \%$ & $0.351^{\mathrm{a}}$ & $55.6 \%$ & $0.487 \mathrm{a}$ & $52.8 \%$ & $0.346^{\mathrm{a}}$ \\
\hline Coagulation necrosis sign & $91.3 \%$ & $7.9 \%$ & $<0.001^{a}$ & $7.4 \%$ & $<0.001^{a}$ & $8.3 \%$ & $<0.001^{a}$ \\
\hline
\end{tabular}

Col \%, column-wise percentages; * malignancy vs benign; ${ }^{\circ}$ malignancy vs tuberculosis; \#malignancy vs sarcoidosis. aChi Square test; bFischer exact test.

Table 3. EBUS sonographic features analysis.

\begin{tabular}{|c|c|c|c|c|c|}
\hline Features on EBUS USG & Sensitivity (\%) & Specificity (\%) & PPV (\%) & NPV (\%) & Diagnostic accuracy (\%) \\
\hline Heterogeneous echogenicity & 65.2 & 66.7 & 41.7 & 84.0 & 66.27 \\
\hline Round shape & 78.26 & 20.63 & 26.47 & 72.22 & 38.37 \\
\hline Absence of CHS & 73.39 & 57.14 & 38.63 & 85.71 & 61.62 \\
\hline Distinct margins & 86.95 & 36.5 & 33.33 & 88.46 & 50 \\
\hline Small axis $>10 \mathrm{~mm}$ & 91.3 & 7.9 & 26.58 & 71.48 & 30.23 \\
\hline CPDI grade 2-3 & 65.21 & 46.03 & 30.61 & 78.37 & 51.16 \\
\hline Coagulation necrosis sign present & 91.3 & 92.0 & 80.7 & 96.11 & 91.86 \\
\hline
\end{tabular}




\section{Discussion}

This is a prospective study for assessing the utility of radiological features of endobronchial ultrasonography in differentiating malignant and benign lymph nodes conducted at a tertiary care referral centre.

Of all the ultrasound features, coagulation necrosis sign was the only characteristic having high sensitivity, specificity and diagnostic accuracy to differentiate malignant from benign lymph nodes. These findings were consistent with previous study done by Fujiwara et al. [6]. This study had enrolled 487 patients and studied 1061 lymph nodes had shown diagnostic accuracy $86 \%$. The sensitivity and specificity were $69.4 \%$ and $92.6 \%$, respectively [6]. The presence of coagulation necrosis signifies the absence of vascularity in the centre as the tumour enlarges in size and hence its presence indicates a rapidly growing cell mass where the vascular structure is not able to keep pace with the cell growth. Since, our study and a similar study have shown high diagnostic accuracy, this feature seems to be a reliable indicator and the one which can be explored further to distinguish between malignant versus benign aetiology of lymph nodes. The other characteristics studied namely distinct margins, round shape; absence of central hilar structure had high sensitivity but low specificity and hence low diagnostic accuracy. These findings are again similar to that of previous studies described in literature [6-8].

Heterogeneous echogenicity had sensitivity and specificity both of $65 \%$ in present study. Fujiwara et al. had shown sensitivity and specificity and diagnostic accuracy of $77.3 \%, 86.6 \%$ and $83.9 \%$, respectively [6]. Schmid-Bindert et al. had shown a high sensitivity and high specificity of $84.8 \%$ and $80.5 \%$, respectively [7]. Heterogeneous echogenicity was initially seen in metastasis from medullary thyroid carcinoma due to deposits of amyloid and calcium. It has also been observed that areas of liquefaction necrosis are seen as hypoechoic and areas of fi-

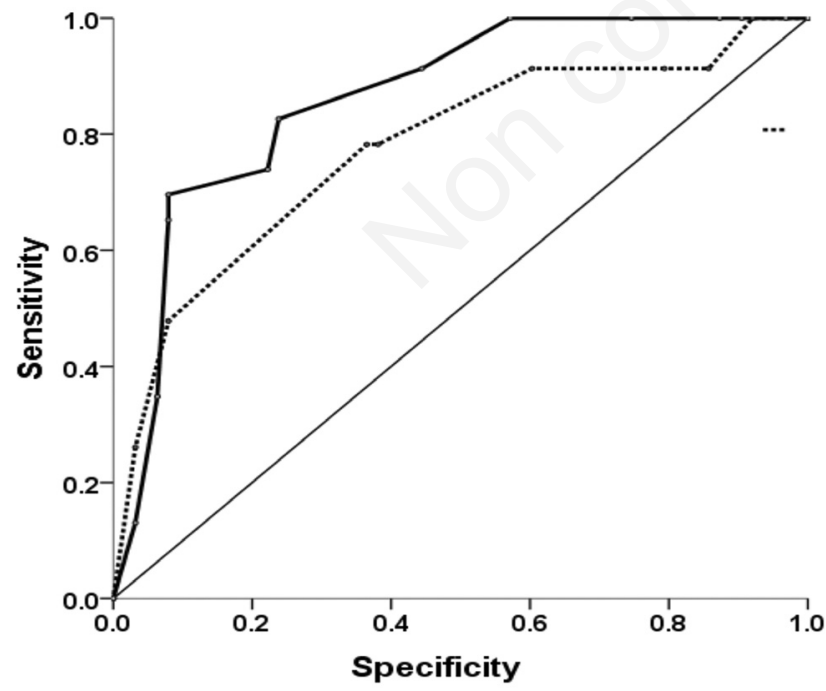

Figure 1. ROC Curve comparing 5 item (Shafiek's) and 7 item score. Dotted line, ROC curve of Shafiek score: AUC $=0.775(95 \%$ CI: 0.68-0.89), $\mathrm{p}<0.001^{\mathrm{a}} \mathrm{b}$; solid line, ROC Curve of 7 item score: AUC $=0.862\left(95 \%\right.$ CI: 0.78-0.94), $\mathrm{p}<0.001^{\mathrm{a}, \mathrm{b}}$; ${ }^{\mathrm{a} u n d e r}$ non-parametric assumption; bnull hypothesis is area $=0.5$. brosis and coagulation necrosis seen as hyperechoic in a lymph node giving it heterogenous appearance. On the other hand, normal, reactive and tuberculous lymph nodes are hypoechoic when compared with adjacent muscles. The small sample size in our study is probably one of the reasons that we are not able to replicate similar results and future studies with a larger sample may further clarify if this feature can definitely be the one with high diagnostic accuracy along with coagulation necrosis sign.

Colour Power Doppler index was only studied by Schmid-Bindert $e t$ $a l$. They have shown sensitivity, specificity and diagnostic accuracy of $50.8 \%, 47.7 \%$ and $49.2 \%$ respectively [7]. Subsequently, they have mentioned that CPDI results have no potential for prediction of malignancy. We have used the same grading for degree of blood flow as used in this study and have got similar findings in our study. As tumour growth and metastasis are closely linked with angiogenesis, it can be rationalised that malignant lymph nodes are more vascular than benign nodes. Normal and reactive lymph nodes either appear avascular or tend to have hilar vascularity, whereas metastatic nodes tend to show peripheral or mixed vascularity, and lymphomatous nodes predominantly demonstrate mixed vascularity. The presence of peripheral vascularity is highly suspicious of malignancy and hence a high CPDI score would be associated with it. Similar results are seen even in inflammatory conditions and therefore this feature cannot be used to distinguish between the two.

Shafiek et al. had given a score combing five features namely shape, margins, echogenicity, size and central hilar structure. They have mentioned that a score of more than 5 had a sensitivity of $78 \%$ and specificity of $86 \%$ (AUC $=0.852 ; 95 \%$ CI: $0.743-0.928 ; p=0.0001$ ) [8]. SchmidBindert $e$ al. had mentioned that the best single criterion to predict malignancy in their study was heterogeneity, and the best negative predictive factor was CHS. The usage of the introduced sum score can be a method to give a higher probability of malignancy: if more than two criteria are present, odds ratio for malignancy is as high as 15.5 [7]. Further studies are recommended to evaluate the potential role of this scoring system. Our study has shown that only presence of coagulation necrosis was associated with high diagnostic accuracy of $91.86 \%$. No other feature had as high a diagnostic accuracy as this. However, a composite score of 5 radiological features as suggested by Shafiek $e t$ al. as well as 7 radiological features as studied here show promise in suggesting malignancy [8].

Our study was a prospective one which included both malignant as well as benign cases of mediastinal and hilar lymphadenopathy. This prevented a recall bias which could have been present in the retrospective studies. Also, the study represented the most common cases of lymphadenopathy in our set up which increases the reliability of our findings. We have used rapid onsite evaluation for all our cases to increase the quality of our results.

The study has its limitations. First of all, the gold standard used here is pathological diagnosis and not surgical exploration of lymph nodes which could result in a verification bias. There were two cases of lung cancer where the sub centimetre non-PET avid lymph node was sampled and was negative. These patients were then referred for surgery but were lost to follow up. Since the surgical data of these lymph nodes is not available, we cannot comment if this could have made any change in our results. The sample size is relatively small and we suggest that a similar study done with a larger sample size may help in confirming the findings of this study. We also suggest that with the advent of elastography, the yield of lymph node sampling can be further increased and this feature can be combined in future studies to distinguish between malignant and benign lymph nodes. 


\section{References}

1. Kumar A, Dutta R, Kannan U, et al. Evaluation of mediastinal lymph nodes using 18F-FDG PET-CT scan and its histopathologic correlation. Ann Thorac Med 2011;6:11-6.

2. Ye T, Hu H, Luo X, et al. The role of Endobronchial ultrasound guided transbronchial needle aspiration (EBUS-TBNA) for qualitative diagnosis of mediastinal and hilar lymphadenopathy: A prospective analysis. BMC Cancer 2011;11:100.

3. Von Bartheld MB, Van Breda A, Annema JT. Complication rate of endosonography (endobronchial and endoscopic ultrasound): A systematic review. Respiration 2014;87:343-51.

4. Ahuja AT, Ying M. Sonographic evaluation of cervical lymph nodes. Am J Roentgenol 2005;184:1691-9.

5. Haner UE. Spaces for creativity and innovation in two established organizations. Creat Innov Manag 2005;14:288-98.

6. Fujiwara T, Yasufuku K, Nakajima T, et al. The utility of sonographic features during endobronchial ultrasound-guided transbronchial needle aspiration for lymph node staging in patients with lung cancer: A standard endobronchial ultrasound image classification system. Chest 2010;138:641-7.

7. Schmid-Bindert G, Jiang H, Kahler G, et al. Predicting malignancy in mediastinal lymph nodes by endobronchial ultrasound: A new ultrasound scoring system. Respirology 2012;17:1190-8.

8. Shafiek H, Fiorentino F, Peralta AD, et al. Real-time prediction of mediastinal lymph node malignancy by endobronchial ultrasound Arch Bronconeumol 2014;50:228-34.

9. Du Rand IA, Barber PV, Goldring J, et al. Summary of the British Thoracic Society Guidelines for advanced diagnostic and therapeutic flexible bronchoscopy in adults. Thorax 2011;66:1014-5.

10. Evison M, Crosbie P, Navani N, et al. How should performance in EBUS mediastinal staging in lung cancer be measured. Br J Cancer 2016;115:e9.

11. Wang L, Wu W, Hu Y, et al. Sonographic features of endobronchial ultrasonography predict intrathoracic lymph node metastasis in lung cancer patients. Ann Thorac Surg 2015;100:1203-9. 